\title{
Coherent lattice vibration of interlayer shearing mode of graphite
}

\author{
Tomobumi Mishina, Kazuki Nitta, and Yasuaki Masumoto \\ Institute of Physics, University of Tsukuba, Tsukuba, Ibaraki 305-8571, Japan
}

(Received 17 April 2000)

\begin{abstract}
We have observed a coherent oscillation signal of the interlayer shearing phonon mode of highly oriented pyrolytic graphite by means of femtosecond pump-probe experiment. The optical polarization dependence reveals a nontrivial phase shift between Rayleigh and Raman components. The detailed analysis of the phase shift clarifies that the Raman component mainly comes from the real part of the dielectric function. The excitation density dependence of the coherent phonon shows the same saturation behavior as that of the electronic response and the generation process of the coherent phonon is attributed to the real excitation of the $\pi-\pi^{*}$ transition.
\end{abstract}

The recent development of femtosecond laser spectroscopy enables the study of the optical phonon in a time domain. The time domain measurement of the lattice vibration offers not only complementary information of the Raman spectrum but also the extended and detailed study of the lattice dynamics. The so-called coherent phonons are observed in many materials and various generation mechanisms are discussed. ${ }^{1-5}$ Interpretative articles have been also published. .,7 $^{-}$

Graphite is one of the standard materials in solid-state physics and its unique property attracts much attention from both fundamental research and industrial application. Therefore, many works have been done on the various properties of graphite in both experimental and theoretical aspects. ${ }^{8-12}$ In ultrafast time-resolved spectroscopy of graphite, the main interest is placed on laser melting and associated phenomena. ${ }^{13,14}$ Although the femtosecond carrier dynamics of graphite has been comprehensively studied, ${ }^{15}$ there were quite a few works on the ultrafast electron and phonon dynamics which were well studied in GaAs and the other semiconductor materials.

In this work, we have observed the coherent phonon oscillation in highly ordered pyrolytic graphite (HOPG). The transient reflectivity measurement gives the coherent phonon signal of interplanar shearing motion whose vibrational energy is about $43 \mathrm{~cm}^{-1} . E_{2 g}$ symmetry of the phonon mode shows its characteristic polarization dependence. From the detailed study of the polarization dependence, we have found a nontrivial phase shift between Rayleigh and Raman components in the reflected probe pulse from the sample. The phase shift is very important in considering the amplitude of the coherent phonon signal. By comparing the phase shift with the numerical calculation, we concluded that the detection process of the coherent phonon is dominated by the off-resonant Raman process. The intensity dependences of the coherent phonon signal and electronic response show the same saturation behavior, which indicates that the coherent phonon is generated by the real excitation of the $\pi-\pi^{*}$ electronic transition.

The sample used in the experiment was a ZYA grade HOPG. Time-resolved pump-probe experiments were performed using a mode-locked titanium-sapphire laser. The time width, the repetition rate, and the center wavelength of the laser pulses were $130 \mathrm{fs}, 82 \mathrm{MHz}$, and $790 \mathrm{~nm}$, respectively. The pump and probe pulses were focused onto the sample with a camera lens and the spot size was $10 \mu \mathrm{m}$. The intensity of the probe pulse was about a fifth of that of the pump pulse. The result of the pump and probe experiment and the schematic atomic displacements for the $E_{2 g}$ interplanar mode are shown in Fig. 1. The inset of Fig. 1 shows a typical pump-probe signal obtained at $293 \mathrm{~K}$ with a pump excitation density of $0.47 \mathrm{~mJ} / \mathrm{cm}^{2}$. The polarizations of the pump and probe pulses crossed each other. The sharp peak structure around the time origin comes from the saturation of the $\pi-\pi^{*}$ electronic transition and relaxes within several picoseconds. ${ }^{15}$ The dashed and solid lines in Fig. 1 are the corresponding time differential signal obtained by the timedelay modulation technique ${ }^{16}$ and the differential signal magnified 1000 times. The modulation amplitude is $0.2 \mathrm{ps}$ and the oscillation signal corresponding to the $E_{2 g}$ interlayer shearing mode appears in the magnification.

In the following we consider the equations describing the interaction between coherent phonons and optical fields. We choose the phonon fields $q_{1}$ and $q_{2}$ so that they have the same transformation properties as those of the functions $x^{2}-y^{2}$ and $x y$ which form a basis of the $E_{2 g}$ irreducible representation. The selection rule for the optical excitation of

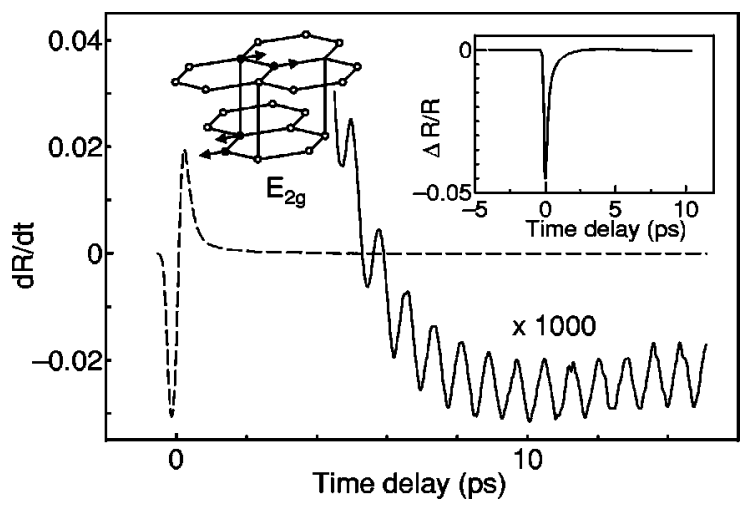

FIG. 1. Coherent oscillation signal of the interlayer shearing phonon mode $E_{2 g}$ of graphite. The dashed line is a whole profile of the pump and probe signal measured by a time-delay modulation technique and the solid line is its 1000 times magnification. The inset shows a corresponding normal pump and probe signal. 
the phonon fields is determined by their symmetric properties irrelevant to the detail of the interaction mechanism. Assuming $\theta$ is an angle between the $x$ axis on the sample surface and the polarization of the pump pulse, the phonon fields impulsively generated by the pump pulse are described as

$$
\left(\begin{array}{l}
q_{1} \\
q_{2}
\end{array}\right)=Q e^{-\alpha z} e^{-\Gamma t} \sin \Omega t\left(\begin{array}{c}
\cos 2 \theta \\
\sin 2 \theta
\end{array}\right),
$$

where $Q$ is the oscillation amplitude proportional to the pump pulse energy, $\Omega$ and $\Gamma$ are the frequency and the damping constant of the phonon, and $\alpha$ is the absorption coefficient of the graphite. Since the phonon dispersion along the $c$ axis of the graphite is very small, ${ }^{17}$ the depth profile of the phonon fields is proportional to that of the pump pulse intensity and $e^{-\alpha z}$ appears in Eq. (1). The penetration depth of the probe light is so thin that the time delay caused by the optical pulse propagation is much shorter than the phonon period. Therefore, we neglect the phase shift and set the phonon oscillation proportional to $\sin \Omega t$. The dielectric constant of graphite is modified by the phonon fields and expressed as

$$
\varepsilon(z, t)=\varepsilon_{b}+\sum_{m} \frac{\partial \varepsilon}{\partial q_{m}} q_{m}(z, t)+\cdots,
$$

where $\varepsilon_{b}$ is the static dielectric constant of the graphite and the Raman tensors in an $x-y$ plane are expressed as

$$
\frac{\partial \varepsilon}{\partial q_{1}}=\left(\begin{array}{cc}
f & 0 \\
0 & -f
\end{array}\right), \frac{\partial \varepsilon}{\partial q_{2}}=\left(\begin{array}{cc}
0 & f \\
f & 0
\end{array}\right) .
$$

We consider the reflection of the probe light by the phonon fields based on the forward and backward scheme. ${ }^{18}$ If we assume the incident probe field to be $\vec{E}_{0} e^{-i \omega t}$, the optical dipole $\vec{P}$ induced by the phonon field is given as

$$
\vec{P}(z, t)=\sum_{m} q_{m}(z, t) \frac{\partial \varepsilon}{\partial q_{m}} \frac{2 n}{1+n} \vec{E}_{0} e^{i(k z-\omega t)},
$$

where $\omega$ is the optical frequency and $n$ and $k$ are the complex refractive index and the complex wave vector derived as $\sqrt{\varepsilon_{b} / \varepsilon_{0}}$ and $n \omega / c$, respectively. The parameters $\varepsilon_{0}$ and $c$ are the dielectric constant and the light velocity in vacuum. By solving the backward equation with the boundary condition, the first-order reflection caused by the phonon perturbation is expressed as

$$
\begin{aligned}
\Delta E_{R}(t) & =-\frac{2}{1+n} \frac{1}{2 c_{b} \varepsilon_{b}} \int_{0}^{\infty} d z^{\prime} \frac{\partial \vec{P}}{\partial t}\left(z^{\prime}, t-\frac{z^{\prime}}{c_{b}}\right) \\
& \approx \frac{-n}{(1+n)^{2} \varepsilon_{b}} \frac{1}{1+i \alpha / 2 k} \sum_{m} \frac{\partial \varepsilon}{\partial q_{m}} q_{m}(z, t) \vec{E}_{0} e^{-i \omega t},
\end{aligned}
$$

where $c_{b}$ is defined as $c / n$. In the approximation, we omit the term proportional to $\Omega / \omega$. If we assume that the polarization of the probe light is parallel to $x$, we can describe the total reflected optical field $\vec{E}_{R}$ using the reflection coefficient $r=(1-n) /(1+n)$ and Eq. $(5)$ as

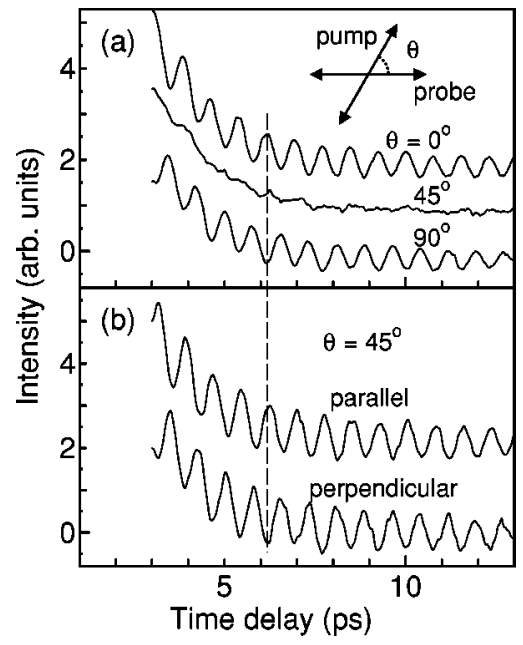

FIG. 2. (a) Polarization dependences of the coherent phonon signal. The oscillation signal shows opposite phases for $0^{\circ}$ and $90^{\circ}$ and disappears at $45^{\circ}$. (b) Recovery of the signal by insertion of the polarizer in front of the probe photodetector.

$$
\vec{E}_{R}(t)=r E_{0} e^{-i \omega t}\left(\begin{array}{l}
1 \\
0
\end{array}\right)+\Delta r E_{0} e^{-i \omega t} e^{-\Gamma t} \sin \Omega t\left(\begin{array}{c}
\cos 2 \theta \\
\sin 2 \theta
\end{array}\right)
$$

where $\Delta r$ is defined as

$$
\Delta r=\frac{d r}{d \varepsilon} \frac{f Q}{1+i \alpha / 2 k} .
$$

The first term and the second term in Eq. (6) are considered as the Rayleigh and Raman components, respectively, depending on their optical frequencies. Here, the value of absorption coefficient $\alpha$ can be taken as twice the imaginary part of $k$. Since the intensity of the reflected optical field $R(t)$ is proportional to the absolute square value $\left|\vec{E}_{R}(t)\right|^{2}$ and $|\Delta r|$ is much smaller than $|r|$, the relative change of the reflection intensity is approximated as

$$
\frac{\Delta R}{R} \approx \frac{|\Delta r|}{|r|} \cos \phi \cos 2 \theta e^{-\Gamma t} \sin \Omega t,
$$

where the angle $\phi$ is given as $\arg (\Delta r / r)$.

The optical polarization dependence of the coherent phonon signal is shown in Fig. 2(a). The angle $\theta$ between pump and probe polarizations changes the oscillation amplitude drastically and the angle dependence is explained by Eq. (8). A similar result is also observed in $\mathrm{LaAlO}_{3} .{ }^{5}$ In order to elucidate the polarization dependence in detail, we checked the polarization property of the probe signal at $\theta=45^{\circ}$. Although Raman and Rayleigh components are present, the oscillation signal disappears due to their crossed polarization as shown in Eq. (6). We inserted the polarizer in front of the photodetector and set its polarization parallel and perpendicular to the pump beam. The obtained signal is shown in Fig. 2(b). The signal recovers and the phase of the oscillation is opposite depending on the polarization, since the Raman and Rayleigh components are mixed by the polarizer.

The heterodyne detection scheme of the Rayleigh and Raman components gives another possibility to measure a relative optical phase between them. The schematic diagram of 

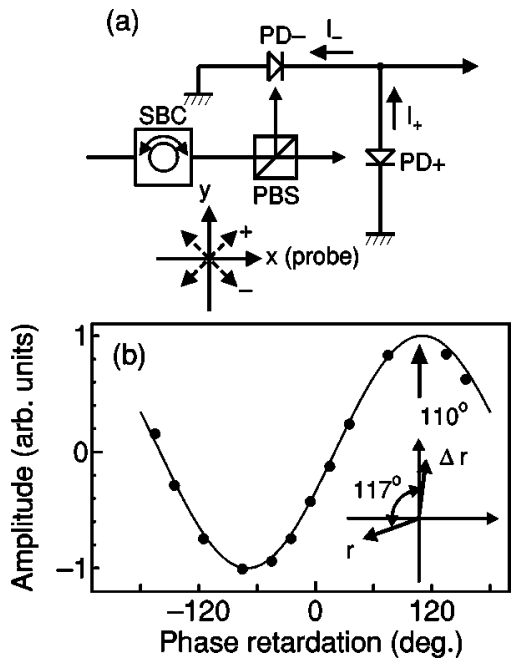

FIG. 3. (a) Schematic diagram of the polarization analyzer. SBC: Soleil-Babinet compensator, PBS: polarization beam splitter, PD: photodiode. (b) Phase retardation dependence of the coherent phonon signal.

the phase measurement is illustrated in Fig. 3(a). If $\theta$ is $45^{\circ}$, the polarizations of these components are orthogonal to each other. By inserting a Soleil-Babinet compensator in the probe path, the phase difference is continuously changed. A polarization beam splitter and two photodiodes are used to extract the pure anisotropic property proportional to the product of the $x$ and $y$ components of the probe beam. The plot of coherent phonon amplitude versus the phase retardation of the Raman component to the Rayleigh component is shown in Fig. 3(b). The accidental phase shift caused by the optical components is compensated. The amplitude reaches its maximum at the phase retardation of $110^{\circ}$. In order to evaluate the phase shift, a diagram of the arguments of $r$ and $\Delta r$ is shown in Fig. 3(b). In the calculation of the arguments, the complex dielectric constant of graphite at $790 \mathrm{~nm}$ is estimated as $4.7+8.6 i$ from the report in Ref. 8, and the factor $f$ of the Raman tensor in Eq. (7) is assumed to be real. The Raman component is advanced by $117^{\circ}$ to the Rayleigh component and the value is very close to the experimental observation. Although the resonant Raman process is expected for the case of the large imaginary part of the dielectric constant, the result clearly shows that the Raman component is dominated by its real part. This means that the off-resonant Raman process works in the detection process of the coherent phonon in graphite.

The temperature dependence of the coherent phonon is shown in Fig. 4. The time-domain signal is shown in Fig. 4(a) and the power spectrum is shown in Fig. 4(b) at $10 \mathrm{~K}$, $160 \mathrm{~K}$, and $325 \mathrm{~K}$. No significant temperature change is observed. The reason for this small temperature dependence is attributable to the lattice structure. The mode corresponds to an entire translation of the graphite layers which is rigidly combined by $\sigma$ electrons. Therefore, the motion of the massive graphite layer requires much energy and is insensitive to the thermal perturbation even at the high temperature where thermal energy exceeds the phonon energy. In our measurement, the center frequency can be clearly determined with an accuracy better than $0.1 \mathrm{~cm}^{-1}$, and the temperature dependence of the frequency is plotted in Fig. 4(c). The plot shows
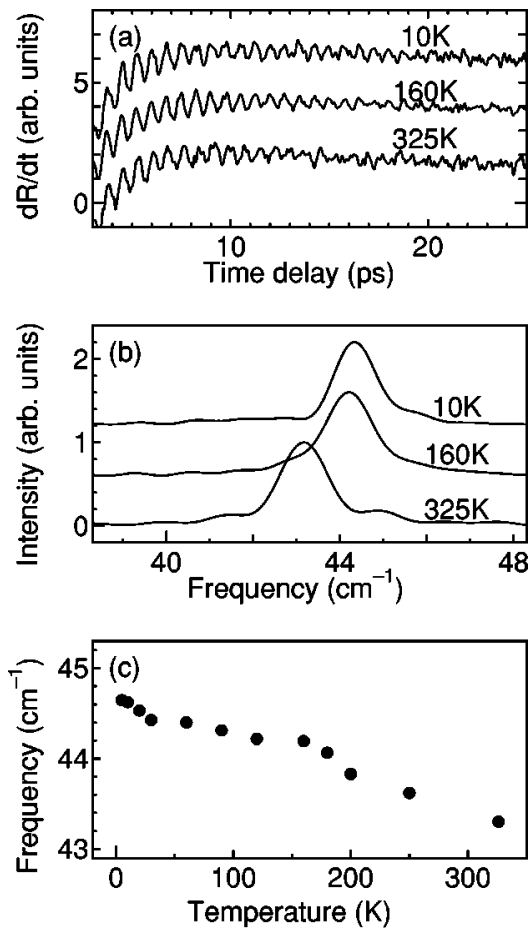

FIG. 4. Temperature dependence of the coherent phonon. (a) Time traces for three temperatures. (b) Corresponding power spectrum. (c) Plot of the phonon frequency versus temperature.

a small but clear redshift. As for the linewidth, the typical value is about $1.0 \mathrm{~cm}^{-1}$, but it shows a deviation of $0.2 \mathrm{~cm}^{-1}$ depending on the sample position. We could not obtain a reliable result on the linewidth and its temperature dependence. This instability may result from the "soft" property of the graphite layer structure.

Figure 5 shows the excitation density dependences. The filled and open circles in Fig. 5(a) represent the amplitude of the phonon signal and the peak value of the electronic re-

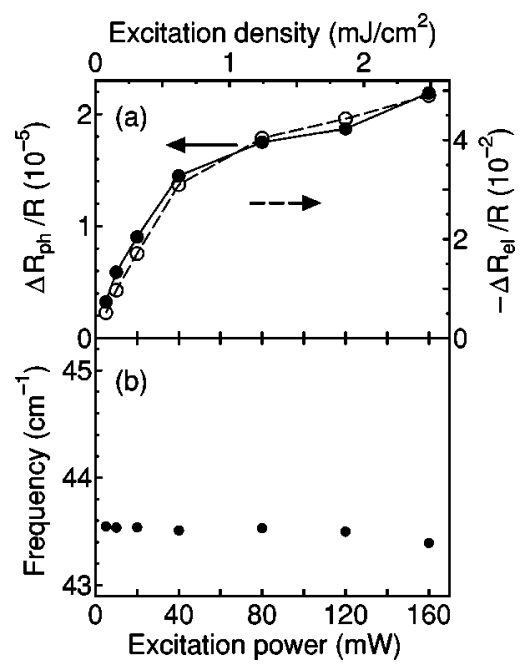

FIG. 5. (a) Excitation dependences of the amplitude of the coherent phonon $\Delta R_{\mathrm{ph}} / R$ and the peak value of the electronic response $-\Delta R_{\mathrm{el}} / R$. Two responses show the same saturation behaviors. (b) Frequency versus excitation density. The frequency is almost constant except for the slight redshift at the high excitation level. 
sponse at the time origin, respectively. The solid and dashed lines are guides for the eyes. The phonon and electronic responses exhibit the same saturation behavior. Figure 5(b) represents the frequency change caused by the excitation density. Except for a slight redshift observed at the high excitation region, the frequency is almost constant and a comparison with Fig. 4(c) denies a significant temperature rise of the sample. Therefore, the saturation behavior comes from a purely electronic origin and the coherent phonon is generated through the $\pi-\pi^{*}$ real optical transition. In addition to the polarization dependence and the impulsive response shown in Eq. (1), this result indicates that the generation mechanism of the coherent phonon is the anisotropic weakening of bonding orbitals which is proposed for $\mathrm{Ge}^{2}$ Since the graphite planes are connected by the covalent bond of $\pi$ electrons, the photoexcitation of the valence electrons preferentially weakens the strength of bonding and causes the polarization dependence. The rapid relaxation of anisotropic hole distribution in momentum space explains the im- pulsive response of the coherent phonon field.

In conclusion, we have observed the coherent oscillation of the interlayer shearing motion in graphite. A detailed study of the polarization dependence shows a nontrivial phase shift between Rayleigh and Raman components of the reflected probe light as large as $110^{\circ}$. By considering the optical process at the graphite boundary, the phase shift clearly shows that the Raman component is dominated by its real part and mainly comes from the off-resonant Raman process. The excitation density dependence of the coherent phonon signal shows a similar saturation behavior to that of the electronic response. The saturation behavior indicates that the coherent phonon is mainly generated by the real excitation of the $\pi-\pi^{*}$ optical transition.

This work was partially supported by the Ministry of Education, Science, Sports and Culture of Japan, Grant-in-Aid for Scientific Research (B), 10440085, 1999 and the "Research for the Future', program JSPS-RFTF97P00106 from the Japan Society for the Promotion of Science.
${ }^{1}$ G.C. Cho, W. Kütt, and H. Kurz, Phys. Rev. Lett. 65, 764 (1990).

${ }^{2}$ T. Pfeifer, W. Kütt, H. Kurz, and R. Scholz, Phys. Rev. Lett. 69, 3248 (1992).

${ }^{3}$ T. Mishina, Y. Masumoto, B. Fluegel, K. Meissner, and N. Peyghambarian, Phys. Rev. B 46, 4229 (1992).

${ }^{4}$ A. Yamamoto, T. Mishina, Y. Masumoto, and M. Nakayama, Phys. Rev. Lett. 73, 740 (1994).

${ }^{5}$ Y. Liu, A. Frenkel, G.A. Garrett, J.F. Whitaker, S. Fahy, C. Uher, and R. Merlin, Phys. Rev. Lett. 75, 334 (1995).

${ }^{6}$ W.A. Kütt, W. Albrecht, and H. Kurz, IEEE J. Quantum Electron. 28, 2434 (1992).

${ }^{7}$ R. Merlin, Solid State Commun. 102, 207 (1997).

${ }^{8}$ E.A. Taft and H.R. Philipp, Phys. Rev. 138, 197 (1965).

${ }^{9}$ R. Ahuja, S. Auluck, J.M. Wills, M. Alouani, B. Johansson, and O. Eriksson, Phys. Rev. B 55, 4999 (1997).

${ }^{10}$ M. Hanfland, H. Beister, and K. Syassen, Phys. Rev. B 39, 12598
(1989).

${ }^{11}$ K. Sinha and J. Menéndez, Phys. Rev. B 41, 10845 (1990).

${ }^{12}$ R. Al-Jishi and G. Dresselhaus, Phys. Rev. B 26, 4514 (1982).

${ }^{13}$ T. Venkatesan, D.C. Jacobson, J.M. Gibson, B.S. Elman, G. Braunstein, M.S. Dresselhaus, and G. Dresselhaus, Phys. Rev. Lett. 53, 360 (1984).

${ }^{14}$ A.M. Malvezzi, N. Bloembergen, and C.Y. Huang, Phys. Rev. Lett. 57, 146 (1986).

${ }^{15}$ K. Seibert, G.C. Cho, W. Kütt, H. Kurz, D.H. Reitze, J.I. Dadap, H. Ahn, M.C. Downer, and A.M. Malvezzi, Phys. Rev. B 42, 2842 (1990).

${ }^{16}$ T. Mishina, Y. Iwazaki, Y. Masumoto, and M. Nakayama, Solid State Commun. 107, 281 (1998).

${ }^{17}$ R. Nicklow, N. Wakabayashi, and H.G. Smith, Phys. Rev. B 5, 4951 (1972).

${ }^{18}$ T. Mishina, Phys. Rev. B 54, 13630 (1996). 